\title{
Agriculture, pesticides and the ecosystem
}

\author{
Rüdiger Pethig, University of Siegen, Germany
}

\section{Introduction}

General equilibrium analysis provided economists with a good understanding of intraeconomy interdependence and a mature and powerful methodology of modeling it. When it became evident that economic activities had detrimental effects on ecological systems (ecosystems, for short), environmental economics developed as a branch of externality theory which, however, focuses on environment-economy interactions often in a rudimentary way only. On the other hand, natural scientists developed models of species interactions in ecosystems (population ecology), and they also study the impact of economic activities on ecosystems but anthropogenic distortions enter their analysis often as exogenous parameter shocks only. It appears, therefore, that in their studies of environment-economy interactions both disciplines, ecology and economics, are biased in opposite directions: Ecologists tend to disregard the complexity of the economic system and economists tend to neglect ecosystem complexity.

In our view, environment-economy interdependence cannot be satisfactorily studied unless both intra-economy and intra-ecosystem interdependence is explicitly modeled and unless repercussions set off in one system by disturbances in the other are captured - including the feedback of these repercussions into the system where the disturbance originated. Natural scientists use to model intra-ecosystem interdependence in dynamic multi-species population models. They apply macro approaches taking populations as basic endogenous variables and hence disregard the interactions of species at the micro level. ${ }^{1}$ In contrast, economic modeling is, in general, microfounded relying on maximizing behavior of firms and consumers.

The present paper aims at applying economic methodology to modeling an ecosystem ${ }^{2}$ with an emphasis on its microfoundation following, to some extent, Hannon (1976), Crocker and Tschirhart (1992) and Tschirhart (2000). It offers, on that basis, a formal analysis of environment-economy interdependence. Our ecosystem submodel of the short period is based on

\footnotetext{
1 This type of modeling is surveyed by Murray (1993) and Brown and Rothery (1993).

2 For a survey and critical assessment of economic approaches to ecosystem analysis see Eichner and Pethig (2001).
} 
the idea that the representative organism of each species behaves 'as if' it maximizes its net energy. Our approach differs from that of Hannon, Crocker and Tschirhart in how the organisms' "production functions" (physiological functions) are specified and in the concept of short-run ecological equilibrium. In contrast to these authors we don't use equilibrating prices but assume, instead, that given the activities of all other organisms each organism acts as if it optimizes its costly offensive and defensive activities (Nash-behavior).

To outline this methodology we develop a three-species model of an ecosystem and link that model to a simple model of the economy with agricultural production and consumption. The three species form a unidirectional non-circular food chain: buzzards feed on mice, mice feed on grain, and grain 'feeds' on solar energy. ${ }^{3}$ There is a fourth species, in fact, the humans, who feed on grain, too. Humans are able to intervene into the ecosystem in three different ways. They can foster the growth (and harvest) of grain by farm labor input; they can use pesticides to diminish the mice population so that mice leave more grain for harvesting; and they can use resources for buzzard habitat maintenance (nature conservation) - with the consequence that buzzards prey more mice. Particular attention will be placed on the derivation of a short-run ecological equilibrium contingent on given levels of economic activities. We then integrate a model of the economy with the ecosystem model with a special focus on the interface of environment-economy interdependence. Both systems are required to settle for an equilibrium simultaneously. From the economist's perspective the ecosystem creates positive and negative externalities (Crocker and Tschirhart, 1992) emerging in agricultural production and in consumer preferences for the ecosystem (green preferences).

Section 2 of the paper elaborates on ecological interdependence as well as on the concept and properties of short-run ecological equilibrium. Section 3 combines the ecosystem model with a model of the economy and characterizes the efficient allocation. Section 4 discusses the inefficiencies of the competitive economy, specifies the types of distortions in the integrated system and briefly investigates some (limited) possibilities to restore efficiency through corrective taxes or subsidies. Section 5 introduces stock-flow relationships and sketches how short-run ecological equilibria are linked to ecosystem dynamics which may or may not drive the ecosystem toward a steady state (long-run ecological equilibrium). Section 6 concludes.

\footnotetext{
${ }^{3}$ This interpretation of the food chain is more allegoric than realistic in nature. Our main objective is to elaborate on a new method of studying ecosystem-economy interdependence.
} 


\section{Ecological interdependence and short-run ecological equilibrium}

To motivate our analysis of short-run ecological interdependence at the micro-level, consider first the standard formal description of population growth,

$$
\frac{n_{v, t+1}-n_{v t}}{n_{v t}}=h_{v t} \quad v=1, \ldots, \bar{v}
$$

With $n_{v t}$ denoting the population of species $v$ in period $t$, equation (1) gives us the rate of population growth in period $t$. Ecological population models use to specify the growth rate of species $v$ in period $t, h_{v t}$, to be dependent on its own population in period $t$, on the populations of some other species and on some vector, $\lambda$, of parameters:

$$
h_{v t}=H^{v t}\left(n_{1 t}, \ldots, n_{\bar{v} t}, \lambda\right) .
$$

In population ecology, predator-prey relationships among species or mutualism are then expressed through the signs of the partials of function $H^{v t}$ with respect to populations. More generally, the functional form of $H^{v t}$ is chosen to reflect more or less well founded empirical hypotheses, but the form is ad hoc in the sense that it does not emerge as an implication of more basic hypotheses in the formal model.

In the next sections we aim at deriving functions of type (2) from species interaction in the short period in which all populations are assumed constant. To keep the exposition simple we envisage an ecosystem in a short period with three species only: buzzards, mice and grain. These species form a unilateral non-circular food chain with buzzards feeding on mice, mice feeding on grain and grain feeding on sunlight. The focus is on representative individual organisms of each species, more specifically, on the net incremental energy these organisms are able to acquire during the period under consideration. These net energies are denoted $g$ for grain, $m$ for mice and $b$ for buzzards. The organisms' net energies are, respectively,

$$
\begin{aligned}
& g=e_{o g} x_{o g}^{d}-e_{g} x_{g m}^{d}-r_{g a} \\
& m=T\left(m_{p}, p\right)\left(e_{g m} x_{g m}^{d}-e_{m} x_{m b}^{d}-r_{m f}-r_{m a}\right), \\
& b=e_{m b} x_{m b}^{d}-r_{b f}
\end{aligned}
$$

where $e_{j}=$ energy per unit biomass of organism $i(i=g, m) ; e_{j}>0$ and constant

$e_{i j}=$ energy intake of predator $j$ per unit of biomass from prey $i$; $e_{i j}>0$ and 


$$
\begin{aligned}
& \text { constant }^{4} \\
& x_{i j}=\text { biomass of prey } i \text { caught by predator } j \\
& r_{i f}=\text { offensive or predation effort of predator } i \text { in terms of own energy spent } \\
& r_{i a}=\text { averting or defensive effort of prey } i \text { in terms of own energy spent } \\
& p=\text { amount of pesticides applied to grain (fields) } \\
& m_{p}=\left(e_{g m} x_{g m}-e_{m} x_{m b}-r_{m f}-r_{m a}\right)=\text { net energy of } m \text { in the absence of pesticides } \\
& T\left(m_{p}, p\right):=\left[1-\delta\left(m_{p}\right) \theta(p)\right] \quad \text { with } \delta\left(m_{p}\right) \begin{cases}=1, & \text { if } m_{p} \geq 0, \\
=-1 & \text { otherwise. }\end{cases}
\end{aligned}
$$

In $T\left(m_{p}, p\right), \theta(p)$ is the fraction of energy of the representative mouse deleted by pesticides $p$ dotted about the grain fields. The function $\theta$ satisfies $\theta(p) \geq 0$ with $\theta(p)=0$ for $p=$ 0 and $^{5} \theta_{p}>0, \theta_{p p} \geq 0$. Note that $T\left(m_{p}, p\right)<0$ if and only if $m_{p}>0$ and $\theta(p)>1$.

Equations (3) implicitly assume that all predators' demands for prey biomass prevails. ${ }^{6}$ The organsism's need of own energy for maintenance (respiration, metabolism, etc.) is accounted for ${ }^{7}$ through the coefficients $e_{i j}$.

The next step is to determine the biomass of prey $i$ caught by predator $j$ as

$$
x_{o g}^{d}=y_{o g}, \quad x_{g m}^{d}=a_{g} y_{g m} \quad \text { and } \quad x_{m b}^{d}=a_{m} y_{m b}
$$

with $^{8}$

$$
\begin{aligned}
& a_{g}=A^{g}\left(n_{g}, n_{m}, r_{g a}\right), \quad a_{m}=A^{m}\left(n_{m}, n_{b}, r_{m a}\right), \\
& y_{o g}=Y^{o g}\left(\ell_{\mathrm{g}}, n_{m}, m, s\right), \quad y_{g m}=Y^{g m}\left(g, n_{g}, n_{m}, r_{m f}\right), \quad y_{m b}=Y^{m b}\left(k, m, n_{m}, n_{b}, r_{b f}\right),
\end{aligned}
$$

\footnotetext{
4 In case of $i=0$ and $j=g$ the intake is not biomass, of course, but sun energy.

5 Subscripts to functional signs denote partial derivatives.

6 This assumption is plausible, in our view. Consistency requires to secure that the intake of prey biomass of all prey organisms equals the outflow of own biomass from all organisms of the prey species. For more details see appendix A.

7 Another way of accounting for maintenance is outlined in appendix A.

8 A minus or plus underneath an argument of a function indicates the sign of the pertinent partial derivative.
} 
where $\left.\left.A^{v}(\cdot) \in\right] 0,1\right]$ with $A^{v}(0)=1, \quad$ and $A_{r r}^{v}>0$. The basic idea behind the equations (4) - (6) is that a prey can reduce the term $a$ by stepping up its averting effort $\left(r_{a}\right)$ while the predator can enlarge the term $y$ and hence its catch of prey biomass, ceteris paribus, by increasing its offensive effort $\left(r_{f}\right)$. Hence the prey biomass taken in by a predator is determined by both the predator's offensive activity $(y)$ and the prey's averting activity $(a)$. In other words, $a_{g}$ and $a_{m}$ reflect the impact of defensive efforts of preys on the predators' preying success, $x$, while $y_{o g}, y_{g m}$ and $y_{m b}$ are the variables under control of the respective predators. 9

The role of populations in (5) and (6) is straightforward. The success of a given averting effort $\left(r_{a}\right)$ is the greater, ceteris paribus, the larger the own population is - because with increasing own population it is the more likely that the predator catches another organism - and the smaller the predator population is - because a predator-prey encounter is then less likely. Prey abundance eases the predator's business of preying (with given predation effort) while an increase in the predator's own population reduces the preying success, ceteris paribus, because the individual predator faces competition from its own kind.

$Y^{o g}$ is the farmers' ecological grain growing 'technology'. He combines seeds, $s$, and farm labor, $\ell_{g}$, to expose the grain to sunlight and to other nutrients like water, minerals etc. available in the ecosystem (but not entering our model). $Y_{m}^{o g}<0$ and $Y_{n_{m}}^{o g}<0$ means that mice impair the cultivation of grain not only through feeding on grain which is captured in $x_{g m}$ but also in other ways. ${ }^{10}$

$Y^{g m}(\cdot)$ is the grain 'harvested' by the representative mouse if grain refrains from averting behavior altogether. $Y^{g m}(\cdot)$ depends on the buzzard's hunting effort, on net mouse energy and on human activity, $k$. We interpret $k$ as a measure of nature conservation benefiting buzzards.

\footnotetext{
9 For example, if $m$ does not undertake any averting effort $\left(r_{m a}=0\right)$, then $a_{m}=1$ and $x_{m b}^{d}=y_{m b}$. Therefore $y_{m b}$ is the buzzard's intake of mice biomass in the absence of defensive activities by mice. If, however, $r_{m a}>0$, then $a_{m}<1$ and the buzzards' intake of mice biomass is $x_{m b}^{d}<y_{m b}$.

10 Mice is probably not a convincing example of an animal species doing harm to plants independent of and/or in addition to feeding. But we find it worthwhile to explore the implications of such a hypothesis (see footnote 1). Anyway, it is easy to 'switch off' this effect simply by setting $Y_{m}^{o g}=Y_{n_{m}}^{o g}=0$.
} 
Increasing $k$ means improving buzzard habitat maintenance to the effect that buzzards become more successful predators ${ }^{11}, Y_{k}^{m b}>0$.

Since populations are kept constant in the short run, we simplify notation by suppressing all population variables in (5) and (6) in the following analysis, but we will 'reactivate' them in section 5. Inserting (4) - (6) in (3) yields

$$
\begin{aligned}
& G^{o}\left(\ell_{g}, m, r_{g a}, y_{g m}\right):=e_{o g} Y^{o g}\left(\ell_{g}, m, s\right)-e_{g} A^{g}\left(r_{g a}\right) y_{g m}-r_{g a} \\
& M^{o}\left(a_{g}, g, p, r_{m a}, r_{m f}, y_{m b}\right):=T\left(m_{p}, p\right)\left[e_{g m} a_{g} Y^{g m}\left(g, r_{m f}\right)-e_{m} A^{m}\left(r_{m a}\right) y_{m b}-r_{m f}-r_{m a}\right], \\
& B^{o}\left(a_{m}, k, m, r_{b f}\right):=e_{m b} a_{m} Y^{m b}\left(k, m, r_{b f}\right)-r_{b f} .
\end{aligned}
$$

There are four different types of arguments in the functions $G^{o}, M^{o}$ and $B^{o}$. First, the organisms' own offensive and/or defensive efforts $(r)$; second, other organisms' predation $(y)$ or defense (a) variables; third, other organisms' net energies; and finally, human activities $k, \ell_{g}$ $p$ and $s$. Obviously, the human or economic activities form links from the economy to the ecosystem. They will be kept constant in the present section but endogenized later and then complemented by links from the ecosystem to the economy.

Recall from (6) that the net energies $g$ or $m$ influence some predator's productivity of hunting. It is plausible to assume that predators take these variables as given, i. e. that they ignore their indirect effect on the net energy of another species. We also assume that each prey takes as given the offensive activities of its predators, and that each predator takes as given the averting activity of its prey. As a consequence, the only variables each organism controls are its own offensive and/or defensive efforts. The principal behavioral assumption is that each organism chooses its efforts as if it maximizes its own net energy - given all other organisms' offensive and/or defensive activities.

The notion that organisms behave 'as if' they maximize their net energy is in line with Hannon (1976), Crocker and Tschirhart (1992), Tschirhart (2000) and others. But while these authors model organisms as price takers the present model assumes Nash behavior in the absence of prices. In other words, we conceive of ecosystem interaction as a non-cooperative

11 We could have modeled the impact of $k$ on buzzards similar as the impact of pesticides on mice - except with opposite sign. Rather than claiming empirical evidence for our procedure the main point we want to make is that human activities can affect the ecosystem in various ways. 
game between the representative organisms of grain, mice and buzzards. ${ }^{12}$ The players and their strategies are listed in table 1:

\begin{tabular}{l|c|c|c} 
Organisms & grain & mice & buzzards \\
\hline Strategies & $a_{g}$ & $a_{m}, y_{g m}$ & $y_{m b}$
\end{tabular}

Table 1: Players and their strategies in the ecosystem game

To determine their own best response to the other players' given strategies the organisms solve, respectively,

$$
\max _{r_{g a}} G^{o}\left(\ell_{g}, r_{g a}, y_{g m}\right), \quad \max _{r_{m a}, r_{m f}} M^{o}\left(a_{g}, g, p, r_{m a}, r_{m f}, y_{m b}\right), \quad \max _{r_{b f}} B^{o}\left(a_{m}, k, m, r_{b f}\right) .
$$

Assuming that the functions $G^{o}, M^{o}$ and $B^{o}$ are strictly concave in $r_{g a},\left(r_{m a}, r_{m g}\right)$ and $r_{b f}$, respectively, the maximizers are determined as functions

$$
r_{g a}=R^{g a}\left(y_{g m}\right), \quad r_{m a}=R_{+}^{m a}\left(y_{m b}\right), \quad r_{m f}=R^{m f}\left(a_{g}\right), \quad r_{b f}=R_{+}^{b f}\left(a_{m}\right) .
$$

We insert the optimal efforts (8) into (5) and (6) to obtain the best responses

$$
\begin{aligned}
& a_{g}=A^{g}\left[R^{g a}\left(y_{g m}\right)\right], \quad y_{g m}=Y^{g m}\left[g, R^{m f}\left(a_{g}\right)\right], \\
& a_{m}=A^{m}\left[R^{m a}\left(y_{m b}\right)\right], \quad y_{m b}=Y^{m b}\left[k, m, R^{b f}\left(a_{m}\right)\right] .
\end{aligned}
$$

Since our model describes a unilateral food chain, it is not surprising that the game disintegrates into two subgames specified in (9a) and (9b). A Nash equilibrium of these subgames consists of strategies $\left(a_{g}^{*}, y_{g m}^{*}\right)$ and $\left(a_{m}^{*}, y_{m b}^{*}\right)$ determined by solving the two equations in (9a) and $(9 b)$, respectively. Total differentiation reveals that there are (equilibrium) functions $\bar{A}^{g}, \bar{A}^{m}, \bar{Y}^{g m}$ and $\bar{Y}^{m b}$ such that

$$
a_{g}^{*}=\bar{A}^{g}(g), \quad y_{g m}^{*}=\bar{Y}^{g m}(g), a_{m}^{*}=\bar{A}^{m}(k, m), \quad y_{m b}^{*}=\bar{Y}^{m b}(k, m) .
$$

12 A game in normal form requires to specify the players, their strategies and their payoffs functions which map strategy profiles into payoffs. In the game under consideration, players and their strategies are well defined and payoffs are net energies. But note that the equations (7) do not represent standard payoff functions since the domain of contains payoffs of other players, among other variables. 
The signs of the partial derivatives in (10) are unambiguous except for $\bar{Y}_{g}^{g m}>0$ and $\bar{Y}_{m}^{m b}>0$. To see this, consider $y_{g m}$ from (9a) and $a_{g}^{*}$ from (10) to write: $y_{g m}=\bar{Y}^{g m}(g)=$. $=Y^{g m}\left\{g, R^{m f}\left[\bar{A}^{g}(g)\right]\right\}$. Total differentiation yields, after some rearrangement of terms,

$$
Y_{g}^{g m}=\underset{+}{Y_{+}^{g m}}+\underset{+}{Y_{+}^{g m}} \cdot R_{-}^{m f} \cdot \bar{A}_{g}^{g} .
$$

To interpret (11) suppose the net energy of grain is increased. Then grain steps up its defensive effort $\left(\bar{A}_{g}^{g}<0\right)$ which has a negative but indirect effect on mice predation productivity. On the other hand, due to $Y_{g}^{g m}>0$ from (6), increasing grain energy has a direct positive effect on mice predation. We consider it plausible that the positive direct effect overcompensates the negative indirect effect. ${ }^{13}$

We proceed to determine the equilibrium net energies by combining (7) with (8) and (10):

$$
\begin{aligned}
& g=G^{o}\left\{\ell_{g}, R^{g a}\left[\bar{Y}^{g m}(g)\right], \bar{Y}^{g m}(g)\right\}, \\
& m=M^{o}\left\{g, p, R^{m a}\left[\bar{Y}^{m b}(k, m)\right], R^{m f}\left[\bar{A}^{g}(g)\right], \bar{Y}^{m b}(k, m)\right\}, \\
& b=B^{o}\left\{\bar{A}^{m}(k, m), k, m, R^{b f}\left[\bar{A}^{m}(k, m)\right]\right\} .
\end{aligned}
$$

The net energies $(g, m, b)$ satisfying (12) constitute a short-run ecosystem equilibrium. In what follows we transform (12) in order to determine each equilibrium net energy as a function of the economic activities $\left(k, \ell_{g}, p, s\right)$, and to specify, at the same time, how the equilibrium net energies respond to exogenous changes of economic activities. It is convenient to proceed in two steps: First we transform each equation in (12) such that the respective species' net energy does not appear on both sides of the equation anymore. As shown in appendix B the system of equations (12) is equivalent to

$$
\begin{gathered}
g=\hat{G}\left(\ell_{g}, m, s\right), \\
+-+ \\
m=\hat{M}\left(\begin{array}{c}
g, k, p), \\
+-+
\end{array}\right.
\end{gathered}
$$

13 The capacity of plants to discourage their predators from feeding on them is small if not even zero. We introduced the assumption $A_{r}^{g}<0$ primarily to demonstrate the generic structure of the food chain model. $\bar{Y}_{m}^{m b}$ is 


$$
\begin{gathered}
b=\hat{B}(k, m) . \\
++
\end{gathered}
$$

The signs of the partials in (13) are clearcut except for $\hat{M}_{g}, \hat{B}_{k}$ and $\hat{B}_{m}$. The signs assigned to these three partials in (13) result from assuming that the offensive activities respond to the variables $g, k$ and $m$, respectively, stronger than the defensive variables. $\hat{M}_{g}>0$ presupposes, in addition, $\theta(p) \leq 1$ in case of $m_{p}>0$. More details are presented in appendix B.

It remains to determine the reduced form of (13). For that purpose we differentiate (13b) and (13c) to obtain

$$
\begin{aligned}
& d g=\frac{1}{1-\hat{G}_{m} \hat{M}_{g}}\left[\hat{G}_{+} d \ell_{g}+\hat{G}_{m} \hat{M}_{k} d k+\hat{G}_{m} \hat{M}_{p} d p\right], \\
& d m=\frac{1}{1-\hat{G}_{m} \hat{M}_{g}}\left[\begin{array}{c}
\left.\hat{M}_{g} \hat{G}_{\ell_{g}} d \ell_{g}+\hat{M}_{k} d k+\hat{M}_{p} d p\right] . \\
-
\end{array}\right.
\end{aligned}
$$

From this information we infer that there are functions $G$ and $M$ such that

$$
\begin{gathered}
g=G\left(\begin{array}{c}
\left.k, \ell_{g}, p, s\right):=\hat{G}\left[\ell_{g}, \hat{M}(g, k, p), s\right], \\
++++
\end{array}\right. \\
m=M\left(k, \ell_{g}, p, s\right):=\hat{M}\left[\hat{G}\left(\ell_{g}, m, s\right), k, p\right] . \\
++-+
\end{gathered}
$$

Finally, we combine (13a) and (14b) to obtain

$$
b=B\left(k, \ell_{g}, p, s\right):=\hat{B}\left[k, M\left(k, \ell_{g}, p, s\right)\right]
$$

In (14c) we have $\partial B / \partial k=: B_{k}=\hat{B}_{k}+\hat{B}_{m} M_{k}$. By setting $B_{k}>0$ we assume (again) that the positive direct effect of buzzard habitat maintenance dominates the negative indirect effect.

The short-run ecosystem equilibrium and the impact of 'shocks' from the economic system on that equilibrium can be conveniently illustrated by recurring to the functions $\hat{G}, \hat{M}$ and $\hat{B}$ from (13). In figure $1\left(b_{0}, g_{0}, m_{0}\right)$ is the solution to (13) for given $k=k_{0}, \ell_{g}=\ell_{g 0}, p=p_{0}$ and $s=s_{0}$.

given by an expression analogous to (11). In contrast to grain, averting behavior of mice is certainly empirically significant. We find it (again) realistic that the indirect effect is of second order only. 
Figure 1: Short-run ecological equilibrium

Figure 1 also shows the impact on the ecological system of increasing the use of pesticides from $p_{0}$ to $p_{1}>p_{0}$. The point of intersection of the curves $\hat{G}(\cdot)$ and $\hat{M}(\cdot)$ shifts from $Q_{0}$ to $Q_{1}$ implying that using more pesticides does not only hurt mice but also buzzards: The net energy of organism $m$ shrinks from $m_{0}$ to $m_{1}$ and that of organism $b$ shrinks from $b_{0}$ to $b_{1}$. The shift from $Q_{o}$ to $Q_{1}$ leaves the representative mouse with reduced but still positive net energy whereas the net energy of the representative buzzard becomes negative $\left(b_{1}<0\right)^{14}$. Note also that the additional use of pesticides increases grain net energy. ${ }^{15}$

Another interesting information is attained through comparative static analysis as follows: We start again with an initial equilibrium for given $\left(k_{0}, \ell_{g 0}, p_{0}, s_{0}\right)$. But now we leave $\ell_{g 0}$ and $p_{0}$ unchanged and improve, instead, nature conservation from $k_{0}$ to $k_{1}>k_{0}$. In figure 1 , $k_{1}$ has been chosen for simplicity such that the new $\hat{M}$-curve intersects the $\hat{G}$-curve in $Q_{1}$ (as before, when $p$ rather than $k$ was increased, c. p.). Hence the impact on grain is the same as in case of increasing $p$, but the buzzard net energy is still positive. In figure 1 , it even increased $^{16}$ from $b_{0}$ to $b_{2}$. Our model thus demonstrates that farmers have at their disposal two different strategies for enhancing farming productivity: fighting against nature (pesticides) or collaborating with nature (buzzard habitat maintenance). Provided that farmers are able and/or willing to take both options into account (which cannot be taken for granted; see below) their choice will depend on comparative costs.

In view of (14) our results on short-run ecological equilibrium and its properties are now summarized in

14 As will be shown in section $5, b_{1}<0$ translates into a declining buzzard population.

15 This effect can be traced back to the assumption $x_{m b}^{d}<y_{m b}$ in (6). If $r_{m a}>0$ one has $a_{m}=1$ so that the $\hat{G}$ curve is vertical in figure 1.

16 Recall, however, from (14c) that $B_{k}>0$ requires the direct effect of $k$ on $\hat{B}$ to dominate the indirect effect $\hat{B}_{m} \hat{M}_{k}$. To illustrate the alternative case, $B_{k}<0$, in figure 1 assume that the dashed line representing function $\hat{B}\left(m, k_{1}\right)$ is drawn sufficiently close to but still below the solid line depicting the graph of $\hat{B}\left(m, k_{0}\right)$. Then we would have $b_{2}<b_{0}$, hence $B_{k}<0$. 


\section{Proposition 1:}

(i) For any given economic activities $\left(k, \ell_{g}, p, s\right)$ there is a unique short-run ecological equilibrium.

(ii) Suppose one of the economic activities is stepped up, ceteris paribus. Then

- an increase in farm labor input $\left(\ell_{g}\right)$ and grain seed $(s)$ benefits all species;

- nature conservation (support for buzzards) ( $k$ ) has a positive effect on grain, a negative effect on mice and an ambiguous effect on buzzards;

- $\quad$ an increased use of pesticides ( $p$ ) benefits grain but hurts mice and buzzards.

It conforms to our intuition that all farming activities, $k, \ell_{g}, p$ and $s$ boost the growth of grain, but it is less intuitive that in (14b) and (14c) farm labor and seed also foster mice and buzzards. In the real world, farming may reduce and/or deteriorate the habitat of mice and buzzards. If that observation were included in our formal model, farm labor and seed would turn out to be less beneficial to the ecosystem.

\section{Efficient farming in the integrated ecosystem-economy model}

In the previous section we investigated the short-run ecological equilibrium, and we provided the interface of ecosystem-economy interdependence via the economic activities $\left(k, \ell_{g}, p, s\right)$. We also demonstrated how (parametric) changes of these economic activities affect the ecosystem. Now we turn to ecological-economic interaction by developing a simple model of the economy with its links to the ecosystem.

The purpose of grain farming is to harvest the entire grain biomass for (human) consumption. ${ }^{17}$ Hence function $G$ in (14a) represents the production function for grain. To ease the exposition we assume that a constant amount of harvested grain is set aside in each period to be used as seed for growing grain in the next period: $s=\bar{s} .18$ Pesticides and nature conservation are assumed be produced with labor input $\ell_{p}$ and $\ell_{k}$, respectively, according to the linear functions

\footnotetext{
17 For simplicity we dispense with modeling grain as an intermediate good to be transformed into final consumer goods say 'bread'.

18 In a more encompassing approach the amount of seed to be retained would be included in the social planer's or the farmers' intertemporal optimization calculus.
} 


$$
\ell_{p}=c_{p} p \quad \text { and } \quad \ell_{k}=c_{k} k \quad\left(c_{p}, c_{k}>0 \text { and constant }\right)
$$

There are $n_{c}$ consumers with utility

$$
\begin{gathered}
u_{i}=U^{i}\left(b, g_{i}, \ell_{i}, m, n_{b}, n_{m}\right) \\
++-+++
\end{gathered} \quad i=1, \ldots, n_{c}
$$

where $\ell_{i}$ is consumer i's endogenous labor supply and $g_{i}$ is his or her consumption of grain. The individual consumer considers the net energies of mice and buzzards as given. But he or she needs not be indifferent with respect to the state of the ecosystem as represented by $g, m$, $n_{b}$ and $n_{m}$. It is conceivable that for $v=m, b, n_{b}, n_{m}$ the marginal utility $U_{v}^{i}$ is zero, positive or negative. We will focus on green preferences, defined by $U_{v}^{i}>0$ for $v=m, b, n_{b}, n_{m}$ and compare this scenario with one where consumers don't care about the ecosystem: $U_{v}^{i}=0$ for $v$ $=m, b, n_{b}, n_{m}$. It should be emphasized, however, that the consumers' positive evaluation of mice and buzzards is not meant to reflect just the esthetics of wildlife as a matter of personal taste but rather relates to important services of nature for human health, recreation biodiversity benefits etc.

The model of the economy is completed by introducing the aggregate constraint for labor,

$$
\sum_{i=1}^{n_{c}} \ell_{i} \geq \ell_{g}+\ell_{k}+\ell_{p}
$$

We restrict our exposition to the case of identical consumers. With this simplification we invoke (14), (15) and (17) to rewrite (16):

$$
u=U\left[B\left(k, \ell_{g}, p, \bar{s}\right), \frac{G\left(k, \ell_{g}, p, \bar{s}\right)-g(\bar{s})}{n_{c}}, \frac{\ell_{g}+c_{k} k+c_{p} p}{n_{c}}, M\left(k, \ell_{g}, p, \bar{s}\right), n_{b}, n_{m}\right]
$$

where $\mathrm{g}(\bar{s})$ is the grain energy needed for retaining the quantity $\bar{s}$ of grain seed to be used in the next period. To characterize an efficient allocation we maximize $U$ with respect to $k, \ell_{g}$ and $p$. Assuming that the functions $G, M$ and $B$ are concave, an interior solution is characterized by the first order conditions 19

$$
\underset{+}{G_{k}}+n_{c} W_{m g} M_{k}+n_{c} W_{b g} B_{k}=-W_{\ell g}
$$

\footnotetext{
19 Corner solutions are ignored in (18). They may be relevant, however, since it may be optimal in some cases to use no pesticides at all.
} 


$$
\begin{gathered}
G_{\ell_{g}}+n_{c} W_{m g} M_{\ell_{g}}+n_{c} W_{b g} B_{\ell_{g}}=-c_{k} W_{\ell g}, \\
+ \\
+
\end{gathered}
$$

where $W_{v g}:=\frac{U_{v}}{U_{g}}$ for $v=b, \ell, m$ is the marginal willingness to pay for $V$ in terms of grain. In (18) the terms $n_{c} W_{m g} M_{v}$ and $n_{c} W_{b g} B_{v}$ for $v=k, \ell_{g}, p$ are aggregate marginal values consumers attach to the economic activity $v$ for its impact on mice and buzzards, respectively. These terms are summation conditions as in Samuelson's well-known rule for the efficient allocation of pure public goods. Hence (18) demonstrates that for an allocation of the economic activities $k, \ell_{g}$ and $p$ to be efficient it is necessary to consider not only their direct productivity effect but also account for the indirect marginal benefits and costs generated by these economic activities through their impact on the ecosystem. In view of this interpretation, the left sides of (18) represent total direct and indirect (net) benefits of activity $v$ and the right side shows marginal labor costs (all in terms of grain). The information (18) is summarized in

\section{Proposition 2:}

(i) Suppose, consumers are indifferent with respect to the ecosystem $\left(W_{m g}=W_{b g}=0\right)$. Then it is efficient to use every input in growing grain such that its marginal productivity equals its marginal cost (in terms of grain). If the marginal productivity falls short of marginal cost, it is efficient not to use the input at all.

(ii) Suppose, consumer preferences are green $\left(W_{m g}, W_{b g}>0\right)$.

$(\alpha)$ It is not efficient to use pesticides unless their marginal productivity $($ at $p=0)$ is sufficiently larger than marginal production cost of pesticides to account for its negative side effects on mice and buzzards.

( $\beta)$ Efficient buzzard habitat maintenance may be at about the same scale as in case consumers don't care about the ecosystem because its positive effect on buzzards is accompanied by a negative side effect on mice. If $W_{b g}>0$ and $W_{m g} \leq 0$, it may be efficient to foster buzzards, even if marginal productivity $G_{k}$ at $k=0$ falls short of marginal labor cost. 
Even though (18) does not allow for a straightforward comparison of allocative efficiency in economies with and without green preferences, its thrust is that the greening of preferences leads to increased farming, reduced use of pesticides and an ambiguous change in buzzards habitat care.

\section{Competitive markets and taxes}

We envisage a perfectly competitive economy with markets for pesticides, grain and labor, and we denote market prices by $q:=\left(q_{k}, q_{\ell}, q_{p}\right)$. There is no market for nature conservation. As in the last section we keep grain seed constant $(s=\bar{s})$ and hencesuppress the market for seed altogether. We also introduce taxes $\tau:=\left(\tau_{k}, \tau_{\ell}, \tau_{p}\right)$ consisting of a tax $\tau_{k}$ on nature conservation, a tax $\tau_{\ell}$ on farm labor and a tax $\tau_{p}$ on pesticides. Tax rates are not signconstrained (hence may turn out to be subsidies).

Recall that the production function for grain $G$ from (14a) implicitly captures all ecological interactions relevant for farming. It was appropriate to have employed this function for characterizing allocative efficiency in the previous section. But in a decentralized market economy farmers may ignore some or all ecosystem interdependence with an impact on farming. To account for incomplete ecosystem information of farmers in a stylized way we distinguish three different types of farmers:

- Farmers who neither use pesticides nor care for the buzzard habitat and who take as given the damage mice inflict on their crop are called ignorant farmers. They consider $g=\hat{G}\left(\ell_{g}, m, \bar{s}\right)$ from (13a) as their relevant grain production function.

- Farmers who know about and take advantage of the productivity enhancing effect of pesticides but who do not care about the buzzard habitat are referred to as conventional farmers. These farmers may disregard nature conservation either because they are ignorant about the implied productivity effect or because habitat maintenance is beyond their control due to limited property rights since the grain fields they own are only a small segment of the buzzards' habitat. Conventional farmers take $g=G\left(k \equiv 0, \ell_{g}, p, \bar{s}\right)$ from (14a) as their grain production function. 
- Farmers who have a full understanding of the grain production function G from (14a) and are also able to take both pesticides and habitat care into account are called green farmers. They use the 'correct' production function $g=G\left(k, \ell_{g}, p, \bar{s}\right)$ from (14a).

These three types of farmers constitute three different economic scenarios each of which has two sub-scenarios depending on whether consumer preferences are green or not. We will not investigate all these scenarios in detail but it appears worthwhile to offer some discussion of their efficiency properties and comparative performance. To reduce complexity we will restrict our attention to integrated ecosystem-economy models in which positive values of nature conservation, farm labor and pesticides are efficient (as implied by (18)).

Green farming (scenario 1). The grain production function is (14) and hence farmers solve the problem

$$
\underset{\left(k, \ell_{g}, p\right)}{\operatorname{Maximize}} q_{g} G\left(k, \ell_{g}, p, \bar{s}\right)-\left(q_{\ell}+\tau_{\ell}\right) \ell_{g}-\left(q_{\ell}+\frac{\tau_{k}}{c_{k}}\right) c_{k} k-\left(q_{p}+\tau_{p}\right) p .
$$

For an interior solution the first order conditions are

$$
q_{k} G_{k}-\tau_{k}=c_{k} q_{\ell}, \quad q_{g} G_{\ell_{g}}-\tau_{\ell}=q_{\ell}, \quad q_{p} G_{p}-\tau_{p}=c_{p} q_{\ell}
$$

The representative consumer solves the Lagrange problem

$$
L=U\left(b, \frac{g-g(\bar{s})}{n_{c}} \ell, m, n_{b}, n_{m}\right)+\lambda\left(q_{\ell} \ell+\rho-\frac{g-g(\bar{s})}{n_{c}}\right),
$$

taking $m$ and $b$ as given. $\rho$ denotes total tax revenue recycled to the consumer in a lump sum fashion. In case of an interior solution the consumer's optimality condition is

$$
-W_{\ell g}=q_{\ell}
$$

Now we combine (22) and (20) to compare the result with (18).

Proposition 3: Consider an economy with green farmers (scenario 1).

(i) If consumer preferences are not green (scenario la) the competitive market allocation is efficient.

(ii) If consumer preferences are green (scenario $1 \mathrm{~b}$ ) the competitive market allocation is efficient, if and only if it is supported by tax/subsidy rates 


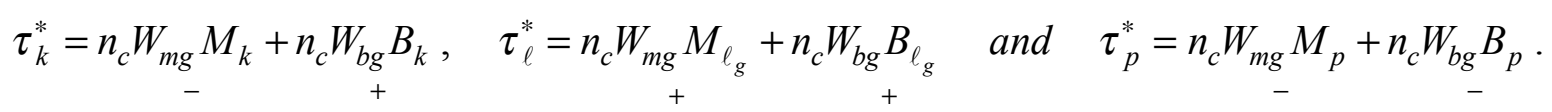

$\tau_{\ell}^{*}$ is a subsidy, $\tau_{p}^{*}$ is a tax and $\tau_{k}^{*}$ may be either a subsidy or a tax or zero.

In scenario 1a the ecosystem depends unilaterally on the economy, and farmers take that linkage fully into account. In contrast, ecosystem disturbances through farming feed back into the economy in case of green preferences (scenario $1 \mathrm{~b}$ ). While the necessity of taxing pesticides was to be expected in the latter scenario, it is less obvious that efficiency requires to subsidize farm labor. As observed in the context of proposition 1, the model appears to overestimate the ecological value of farm labor because it ignores the ecological opportunity costs of growing grain.

Conventional farming (scenario 2). We replace the farmers' maximization problem (19) by $\underset{\left(\ell_{g}, p\right)}{\operatorname{Maximize}} q_{g} G\left(k \equiv 0, \ell_{g}, p, \bar{s}\right)-\left(q_{\ell}+\tau_{\ell}\right) \ell_{g}-\left(q_{p}+\tau_{p}\right) p$. This yields the FOCs $q_{g} G_{\ell_{g}}-\tau_{\ell}=$ $=q_{\ell}$ and $q_{g} G_{p}-\tau_{p}=c_{p} q_{\ell}$, as before, but also implies $q_{k} G_{k}-\tau_{k}>c_{k} q_{\ell}$. Owing to the last inequality conventional farming induces an allocative distortion which is the only cause of inefficiency in scenario 2, if consumer preferences are not green (scenerio 2a). In case of green preferences the production externality of scenario $2 \mathrm{a}$ is augmented by the consumption externalities with regard of mice and buzzards (scenario $2 \mathrm{~b}$ ). Note also that with farmers of the conventional type there does not exist a tax-subsidy scheme $\tau:=\left(\tau_{k}, \tau_{\ell}, \tau_{p}\right)$ to restore efficiency ${ }^{20}$.

On the other hand, if farmers are not able to care for the buzzards habitat themselves, ecological education and enlightenment does not help. Instead, some kind of cooperative arrangement between farmers and the owners of the habitat would be necessary to provide for efficient habitat maintenance. In case such cooperation fails or the habitat is made up of public lands and forests, the government is called for to induce or provide appropriate nature conservation services.

Ignorant farming (scenario 3). In this case, the farmers' maximization problem is

20 The reason is that the solution to (23) subject to $k \equiv 0$ implies $\left.G_{k}\right|_{k=0}>q_{\ell}=-W_{\ell g}$. There is a second-best tax-subsidy scheme $\left(\tau_{g}^{o}, \tau_{p}^{o}\right)$, but our conjecture is that the pertaining quantity of pesticides, $p_{o}$, is greater than the efficient quantity $p *$. 
$\underset{\left(\ell_{g}\right)}{\operatorname{Maximize}} q_{g} \hat{G}\left(\ell_{g}, m, \bar{s}\right)-\left(q_{\ell}+\tau_{\ell}\right) \ell_{g}$. Clearly, growing grain is now severely distorted by two distinct production externalities, since the farmers ignore the impact of both $k$ and $p$ on $m$. The resultant inefficiency is further aggravated when consumer preferences are green. With the tax on farm labor being the only tax instrument left, efficiency cannot be restored, in general.

Casual observation of modern agriculture shows that farmers learnt to take advantage of the productivity effect of pesticides (and use them excessively, at times). Hence the underprovision of nature conservation services of scenario 2 may well be a more serious empirical problem than the failure of using pesticides (if and when it is appropriate) in scenario 3.

The preceding discussion is summed up in

Proposition 4: Consider a general competitive equilibrium in an integrated ecosystemeconomy model.

(i) Without policy intervention, the equilibrium is efficient if and only if ( $\alpha)$ all consumers are indifferent with respect to the state of the ecosystem, $(\beta)$ farmers have a full understanding of ecosystem interdependence on growing grain and $(\gamma)$ the appropriate level of nature conservation is secured either by the farmers themselves or others (e.g. the government).

(ii) If one or both of the last two conditions fail to hold, efficiency cannot be restored with the help of taxes or subsidies on farm labor, pesticides and/or nature conservation services. ${ }^{21}$

If market allocations are inefficient, the comparison of marginal conditions characterizing efficiency on the one hand and market distortions on the other hand doesn't allow for straightforward conclusions about how the inefficient market allocation deviates from the optimum. In particular, we don't know how the net energies of mice and buzzards deviate from their optimum values. Precise answers to these questions would require numerical analysis which is beyond the scope of this paper, however. Our conjecture is that in all scenarios (except the efficient scenario 1a) the no-policy market allocation is characterized by excessive use of pesticides, by too little labor input in farming, by insufficient nature conservation and by too small net energies of mice and buzzards. Grain may be above or below its efficient level.

21 This observation does not imply that other policy instruments couldn't do the job. But the discussion of alternative instruments is beyond the scope of the present paper. 
Up to this point, our integrated ecosystem-economy analysis provided a number of interesting insights and results. But since this paper is primarily about the methodology of integrated ecosystem-economy analysis, it is also necessary to spell out more explicitly the logic of the joint short-run equilibrium in both systems. Clearly, in each of the scenarios defined above, $a$ joint short-run ecosystem-economy equilibrium is constituted by a vector of prices and taxes $(q, \tau)$ and an allocation $\left(g, m, b ; k, \ell_{g}, p, \bar{s}\right)$ such that

- $\quad\left(k, \ell_{g}, p, \bar{s}\right)$ satisfies (19) and is technologically feasible,

- consumers maximize their utility and producers maximize their profits for given $(q, \tau)$ and

- the equations $g=G\left(k, \ell_{g}, p, \bar{s}\right), m=M\left(k, \ell_{g}, p, \bar{s}\right)$ and $b=B\left(k, \ell_{g}, p, \bar{s}\right)$ from (14) hold.

Hence a joint short-run equilibrium requires (i) the simultaneous determination of an equilibrium in both the ecosystem and the economy and (ii) the equilibrium allocation depends on the economic scenario as well as on the tax rates chosen (if any). To restate the observation (ii) in more formal terms we introduce the index $\sigma=1 \mathrm{a}, 1 \mathrm{~b}, 2 \mathrm{a}, 2 \mathrm{~b}, 3 \mathrm{a}, 3 \mathrm{~b}$ to describe the scenario of the economy under consideration and write the joint equilibrium allocation as $\left[G^{*}(\sigma, \tau), M^{*}(\sigma, \tau), B^{*}(\sigma, \tau), K^{*}(\sigma, \tau), L^{*}(\sigma, \tau), P^{*}(\sigma, \tau),\right]$, where

$$
\begin{aligned}
G^{*}(\sigma, \tau) & =G\left[K^{*}(\sigma, \tau), L^{*}(\sigma, \tau), P^{*}(\sigma, \tau)\right], \\
M^{*}(\sigma, \tau) & =M\left[K^{*}(\sigma, \tau), L^{*}(\sigma, \tau), P^{*}(\sigma, \tau)\right], \\
B^{*}(\sigma, \tau) & =B\left[K^{*}(\sigma, \tau), L^{*}(\sigma, \tau), P^{*}(\sigma, \tau)\right] .
\end{aligned}
$$

\section{Ecosystem dynamics and long-run ecological equilibrium}

Up to this section the populations of all species, $n_{g}, n_{m}$ and $n_{b}$, have been set constant. This was an appropriate assumption for the short period but it cannot be maintained, of course, when time is introduced. Now we denote the populations in period $t$ by $n_{v t}$ for $v=g$, $m, b$ and we stick to the simplifying assumption that grain is fully harvested in each period with a constant amount of it being retained for growing grain in the next period. This amounts to assuming $n_{g t} \equiv \bar{n}_{g}(\bar{s})>0$ for all $t$. Hence it is only the populations of mice and buzzards that change in time.

The next step is to relate equilibrium net energies $m_{t}$ and $b_{t}$ to populations. This is done in a stylized way by assuming that $\gamma, \mu$ and $\beta$ is the constant average net energy stored in each 
organism of grain, mice and buzzards, respectively. Consequently, $g_{t} / \gamma, m_{t} / \mu$ and $b_{t} / \beta$ is the average number of new organisms bred in period $t$ by each grain, mouse or buzzard existing at the beginning of period $t$. For example, $m_{t} / \mu=2.34$ means that each mouse living in period $t$ has, on average, 2.34 off-springs (which are assumed to be grown up at the end of period $t$ ). Similarly, $m_{t} / \mu=-0.16$ is interpreted as a situation were the average mouse has no descendents and a $16 \%$ chance not to survive the period. Hence at the beginning of period $t+$ 1 the populations are

$$
n_{g, t+1}:=\left(\frac{0}{\gamma}+1\right) n_{g}(\bar{s})=n_{g}(\bar{s}), \quad n_{m, t+1}:=\left(\frac{m_{t}}{\mu}+1\right) n_{m t} \quad \text { and } \quad n_{b, t+1}:=\left(\frac{b_{t}}{\beta}+1\right) n_{b t}
$$

implying the population growth rates

$$
\frac{n_{g, t+1}-n_{g t}}{n_{g t}}=0, \quad \frac{n_{m, t+1}-n_{m t}}{n_{m t}}=\frac{m_{t}}{\mu} \quad \text { and } \quad \frac{n_{b, t+1}-n_{b t}}{n_{b t}}=\frac{b_{t}}{\beta} .
$$

Comparing (25) to (1) and setting $h_{m t}=m_{t} / \mu$ and $h_{b t}=b_{t} / \beta$ reveals that our entire previous analysis was directed to provide a foundation for the population growth rates. Clearly, $m_{t}$ and $b_{t}$ in (25) are specified by the functions $M^{*}$ and $B^{*}$ from (23). But at this point, it is necessary to recall that in the short period populations entered the analysis of section 2 in (3), (5), (6) and (16) but were suppressed in the subsequent formal analysis for notational convenience. We now need to 'reactivate' the populations $n_{t}:=\left(n_{g t}, n_{m t}, n_{b t}\right)$ as determinants of short-run ecological equilibrium because in the long term these populations are endogenous. In other words, we simply observe that the functions $G^{*}, M^{*}$ and $B^{*}$ from (23) also depend on populations:

$$
g_{t}=G^{*}\left(n_{t}, \sigma, \tau_{t}\right), \quad m_{t}=M^{*}\left(n_{t}, \sigma, \tau_{t}\right), \quad b_{t}=B^{*}\left(n_{t}, \sigma, \tau_{t}\right)
$$

Conceptually, the sign of the derivatives $\partial V^{*} / \partial n_{t}$ for $V^{*}=G^{*}, M^{*}, B^{*}$ from (26) is determined in our model of the short period (as well as the signs of $\partial V^{*} / \partial \sigma$ and $\partial V^{*} / \partial \tau_{t}$ ). But due to the complexities of short-run ecosystem-economy interdependence the net effects of $n_{t}, \sigma$, and $\tau_{t}$ on the equilibrium values of net energies cannot be easily determined. One needs to resort to numerical analysis for specifying the impact of $n_{t}, \sigma$, and $\tau_{t}$ on net energies. In the present paper we content ourselves with the limited qualitative information condensed in (26) and proceed by combining (25) and (26) to obtain 


$$
\frac{n_{m, t+1}-n_{m t}}{n_{m t}}=\frac{M^{*}\left(n_{t}, \sigma, \tau_{t}\right)}{\mu}, \quad \frac{n_{b, t+1}-n_{b t}}{n_{b t}}=\frac{B^{*}\left(n_{t}, \sigma, \tau_{t}\right)}{\beta}
$$

Without numerical specification of the functional forms $G^{*}, M^{*}$ and $B^{*}$ it is impossible to characterize the ecosystem dynamics (27). We observe, however, that since population growth rates depend on human behavior, economic activities and on environmental policy, humans have great influence on how the ecosystem develops over time. In case that for given values of $\bar{s}, \sigma$, and $\tau$ the ecosystem dynamics converge to a steady state, also called long-run ecological equilibrium, the stationary populations $n^{*}:=\left[n_{g}(\bar{s}), n_{m}^{*}, n_{b}^{*}\right]$ are implicitly determined by

$$
G^{*}\left(n^{*}, \sigma, \tau\right)>0 \text { and const. }, \quad M^{*}\left(n^{*}, \sigma, \tau\right)=B^{*}\left(n^{*}, \sigma, \tau\right)=0
$$

\section{Concluding remarks}

We first modeled short-run intra-ecosystem interdependence based on species behavior at the micro level and introduced the concept of short-run ecosystem equilibrium. Then we demonstrated that ecosystem-economy interdependence can be fruitfully studied by linking a standard perfectly competitive economy with our full-fledged (short-run) equilibrium model of the ecosystem. Owing to the interdependence of both systems, the joint equilibrium needs to be simultaneously determined. As a result, intertemporal economic performance depends on how the species populations develop over time. Conversely, the species population dynamics depend on farming styles (ignorant, conventional, green) on consumer valuation of the ecosystem (preferences being green or not) and on ecosystem policies (here only: taxes or subsidies).

If preferences are green, farming and other human activities which have an impact on the ecosystem create vast positive or negative consumption externalities. A rather unexpected result of green preferences is that subsidizing farm labor is efficiency enhancing even though this conclusion may not be robust when ecological opportunity costs of farming are properly accounted for. With our main focus on agriculture we showed that, via ecological food chains, agriculture has an indirect influence on some of those species, exemplified by buzzards in our simple model, that are not directly linked to farming. Moreover, in the light of our analysis the concept of efficient farming needs to be closely reconsidered depending on which and/or how many of the farming-related ecosystem interdependencies farmers take into account. While these insights are not entirely novel, our objective was to derive them in a formal well-defined 
model that explicitly allows to deal with relevant ecological interactions and 'shocks' spreading from one system to the other including feedback effects into the system where they originated.

We emphasized in the introduction that the main contribution of the present paper is to suggest a new methodological approach to the analysis of ecosystem-economy interdependence. Hence our principal focus was on the conceptual procedure rather than on substantial results. Even though some interesting specific conclusions have been reached, important questions remained unanswered especially about the characteristics of ecosystem dynamics and of long-run ecological equilibrium. Among the issues on the agenda of future research is also the question, e. g., under which conditions one would obtain, in the framework suggested here, predator-prey population interactions of the Lotka-Volterra type. In our model, population dynamics with endogenously determined growth rates can probably only be handled in numerical analysis. As is well known, there is a considerable cost of calibration in terms of loss in algebraic generality. But the upside is that one can add much more realistic structure to the model so that it is no longer allegoric (see footnote 3 ) but can be applied and/or tested in realworld case studies.

Another possibly controversial issue is whether it is sensible to model all economic and ecological agents as myopic maximizers, as we did. In our view, maximizing within the time horizon of the short period appears to be appropriate for individual organisms in the ecosystem. But economic agents are forward looking, at least to some extent, and therefore it might be more realistic to conceive of them as intertemporal maximizers. It is not so clear, however, whether assuming rational expectations and maximizing over an infinite time horizon is more realistic than the opposite polar case of myopic maximization in the short period. Anyway, it seems necessary and worthwhile to further follow both lines of modeling, since the comparison promises to give additional insights into the important issue of sustainable development.

When environmental and nature conservation policies are at issue, myopia is definitively not an adequate guideline. In our paper, we did not give adequate attention to such policy issues. We investigated only briefly the potential and limits of welfare improving tax-subsidy schemes applied in the short period (and hence being also myopic) which allowed us to identify such tax policies as a major determinant of population growth rates and hence of ecosystem dynamics, more generally. The few myopic tax policies discussed in the paper only served the role to point out that in most economic scenarios there is scope for efficiency-enhancing environmental and agricultural policies. However, since the relevant policy goals are about 
ecosystem development and sustainability, all serious policies need to focus on the intertemporal development and control of both the ecosystem and the economy. Addressing these issues on the basis the integrated analytical framework suggested here appears to be promising.

\section{References}

Amir, S. (1979), "Economic interpretations of equilibrium concepts in ecological systems", Journal of Social and Biological Structure 2, 293-314

Brown, D., and Rothery, P. (1993), Models in Biology: Mathematics, Statistics and Computing, Wiley \& Sons, Chichester

Crocker, T. D., and Tschirhart, J. (1992), "Ecosystems, externalities, and economies", Environmental and Resource Economics 2, 551-567

Eichner, T., and Pethig, R. (20001), Economic approaches to ecosystem analysis, manuscript

Hannon, B. (1976), "Marginal product pricing in the ecosystem", Journal of Theoretical Biology $56,253-267$

Murray, J. D. (1993), Mathematical Biology, $2^{\text {nd }}$ ed., Springer Verlag, Berlin

Stroebele, W. J., and Wacker, H. (1995), "The economics of harvesting predator-prey systems", Journal of Economics 61, $65-81$

Tschirhart, J. (2000), "General equilibrium of an ecosystem", Journal of Theoretical Biology $203,13-32$

\section{Appendix}

\section{Appendix A: Biomass transactions and energy needed for maintenance}

Rather than using the equations (3) as the basic building block of the model consider

$$
\begin{aligned}
& g=\left(\widetilde{e}_{o g} x_{o g}^{d}-\widetilde{e}_{g} x_{g m}^{s}-\widetilde{r}_{g a}\right)\left(1-\alpha_{g}\right), \\
& m=T\left(m_{p}, p\right)\left(\widetilde{e}_{g m} x_{g m}^{d}-\widetilde{e}_{m} x_{m b}^{s}-\widetilde{r}_{m f}-\widetilde{r}_{m a}\right)\left(1-\alpha_{m}\right), \\
& b=\left(\widetilde{e}_{m b} x_{m b}^{d}-\widetilde{r}_{b f}\right)\left(1-\alpha_{b}\right),
\end{aligned}
$$


where $g, m$ and $b$ are net energy, as in (3) and where the symbols with a 'wiggle' are defined as the corresponding terms without a wiggle in (3). $\alpha_{i}$ denotes the energy used up for maintenance (respiration, metabolism etc.) per unit of (gross) energy, $\left.\alpha_{i} \in\right] 0,1[$. The superscripts $s$ and $d$ refer to amounts of biomass supplied and demanded, respectively.

Denote by $n_{v}$ for $v=g, m, b$ the (constant) population of species $v$, i.e. the number of its organisms in the short period under consideration. Since biomass intake by a predator must equal the biomass outflow from its prey we clearly require

$$
n_{g} x_{g m}^{s}=n_{m} x_{g m}^{d} \text { and } n_{m} x_{m b}^{s}=n_{b} x_{m b}^{d}
$$

It is assumed that all predators' demands prevail. Therefore $x_{g m}^{s}$ and $x_{m b}^{s}$ are substituted in (A1) by $\left(n_{m} / n_{g}\right) x_{g m}^{d}$ and $\left(n_{b} / n_{m}\right) x_{m b}^{d}$, respectively. In addition, we suppress all population variables, since populations are constant in the short run, and we further simplify the notation by setting $e_{i j}:=\widetilde{e}_{i j}\left(1-\alpha_{j}\right), e_{j}:=\widetilde{e}_{j}\left(1-\alpha_{j}\right), r_{i a}:=\widetilde{r}_{i a}\left(1-\alpha_{i}\right)$ and $r_{i f}:=\widetilde{r}_{i f}\left(1-\alpha_{i}\right)$. Thus (A1) and (A2) are transformed into (3) (with populations being dropped to avoid clutter).

\section{Appendix B: Derivation of (13)}

We insert $r_{g a}$ from (8) into (7a): $G^{o}(\cdot)=e_{o g} Y^{o g}\left(\ell_{g}, m, s\right)-e_{g} A^{g}\left[R^{g a}\left(y_{g m}\right)\right] y_{g m}-R^{g a}\left(y_{g m}\right)$. Differentiation yields $\quad d g=e_{o g} \underline{\ell}_{+}^{o g} d \ell_{g}+e_{o g} Y_{-}^{o g} d m+e_{o g} Y_{+}^{o g} d s-\left[\left(e_{g} A_{r}^{g}+1\right) R_{y}^{g a}+e_{g} a_{g}\right] d y_{g m}$. Since $e_{g} A_{r}^{g}+1=0$ is the FOC for maximizing $G^{o}$ with respect to $r_{g a}$, we obtain

$$
g=G^{l}\left(\begin{array}{l}
\left.\ell_{g}, m, s, y_{g m}\right) \\
+++-
\end{array}\right.
$$

(B1) and $y_{g m}$ from (10) readily imply

$$
\begin{gathered}
G\left[\ell_{g}, m, s, \bar{Y}^{g m}(g)\right] \\
+-+\underbrace{++}_{-}
\end{gathered}=\begin{gathered}
\hat{G}\left(\ell_{g}, m, s\right) . \\
+-+
\end{gathered} .
$$

Consider next $r_{m f}$ and $r_{m a}$ from (8) in (7b):

$$
M^{o}(\cdot)=T\left(m_{p}, p\right)\left\{e_{m g} a_{g} Y^{g m}\left[g, R^{m f}\left(a_{g}\right)\right]-e_{m} A^{m}\left[R^{a m}\left(y_{m b}\right)\right] y_{m b}-R^{m a}\left(y_{m b}\right)-R^{m f}\left(a_{g}\right)\right\} .
$$


We differentiate this equation totally and take into account that $e_{m} y_{m b} A_{r}^{m}+1=0$ and $e_{m} a_{g} Y_{r}^{g m}=1$ is implied by maximizing $M^{0}$ with respect to $r_{m f}$ and $r_{m a}$. This yields

$$
\begin{aligned}
d m=\underbrace{m_{p} T_{p}}_{-} d p+T\left(m_{p}, p\right) e_{m g} y_{g m} d a_{g}+T\left(m_{p}, p\right) e_{m g} a_{g}{\underset{+}{g}}_{+}^{g m} d g-\theta_{e m} a_{m} d y_{m b} \text { and hence } \\
\\
\quad m=M^{1}\left(a_{g}, g, p, y_{m b}\right) .
\end{aligned}
$$

Since $\theta_{p}>0$ we clearly have $T_{p}=-m_{p} \delta\left(m_{p}\right) \theta_{p}<0$, but the sign of $M_{a}^{1}$ and $M_{g}^{1}$ depends on the sign of $T\left(m_{p}, p\right)$. Invoking $a_{g}$ and $y_{m b}$ from (10) transforms (B2) into

$$
\begin{aligned}
& m=M^{1}\left[\bar{A}^{g}(g), g, p, \bar{Y}^{m b}(k, m)\right] . \text { Differentiation yields } \\
& d m=\frac{M_{a}^{l} \bar{A}_{g}^{g}+M_{g}^{l}}{1-M_{y}^{l} \bar{Y}_{m}^{m b}} d g+\frac{M_{p}^{l}}{1-M_{y}^{l} \bar{Y}_{m}^{m b}} d p+\frac{M_{y}^{l} \bar{Y}_{k}^{m b}}{1-M_{y}^{l} \bar{Y}_{m}^{m b}} d k .
\end{aligned}
$$

The second an third terms on the right side of (B3) are negative. The numerator of the first term is $M_{g}^{1}+M_{a}^{1} \bar{A}_{g}^{g}$. Analogous to (11) $M_{g}^{1}=T\left(m_{p}, p\right) e_{m g} a_{g} Y_{g}^{g m}$ is the direct effect of grain on mice via the impact of grain on mice preying productivity. On the other hand,

$$
M_{a}^{1} \bar{A}_{g}^{g}=T\left(m_{p}, p\right) e_{m g} y_{g m} \frac{A_{r}^{g} R_{y}^{g a} Y_{g}^{g m}}{1-A_{r}^{g} R_{y}^{g a} Y_{r}^{g m} R_{a}^{m f}}
$$

represents the indirect effect of $g$ on $m$ caused by a growing grain population stepping up its defense. It is plausible to assume that the sign of the net effect is always determined by the sign of the direct effect, $M_{g}^{l}$. Hence

$$
M^{l}\left[\bar{A}^{g}(g), g, p, \bar{Y}^{m b}(k, m)\right]=\hat{M}(g, k, p)
$$

where $\hat{M}_{g}>0$ unless $m_{p}>0$ and $\theta(p)>1$. For convenience of exposition we restrict our further investigation to situations where $\hat{M}_{g}>0$.

Now we plug $a_{m}$ from (10) and $r_{b f}$ from (8) into (7c):

$$
B^{o}(\cdot)=e_{m b} \bar{A}^{m}(k, m) Y^{m b}\left\{k, m, R^{b f}\left[\bar{A}^{m}(k, m)\right]\right\}-R^{b f}\left[\bar{A}^{m}(k, m)\right]
$$

Since $e_{m b} a_{m} Y_{r}^{m b}=1$ holds when $B^{0}$ is maximized with respect to $r_{b f}$, differentiation results in 


$$
d b=e_{m b}\left(y_{m b} \bar{A}_{-}^{m}+a_{m} Y_{+}^{m b}\right) d k+e_{m b}\left(\begin{array}{c}
y_{m b} \bar{A}_{m}^{m}+a_{m} Y_{+}^{m b} \\
Y_{+}
\end{array}\right) d m .
$$

For $v=k, m$, the right side of (B4) is symmetric. $Y_{v}^{m b}$ is the direct and positive effect on buzzard predation productivity while $y_{m b} \bar{A}_{v}^{m}<0$ is the reduction in predation success caused by the mice's defensive response to increases in $v$. It appears plausible, again, to assume that the positive direct effect overcompensates the indirect effect. Hence

$B^{o}\left\{\bar{A}^{m}(k, m), k, m, R^{b f}\left[\bar{A}^{m}(k, m)\right]\right\}=\underset{+}{\hat{B}(k, m) .}$

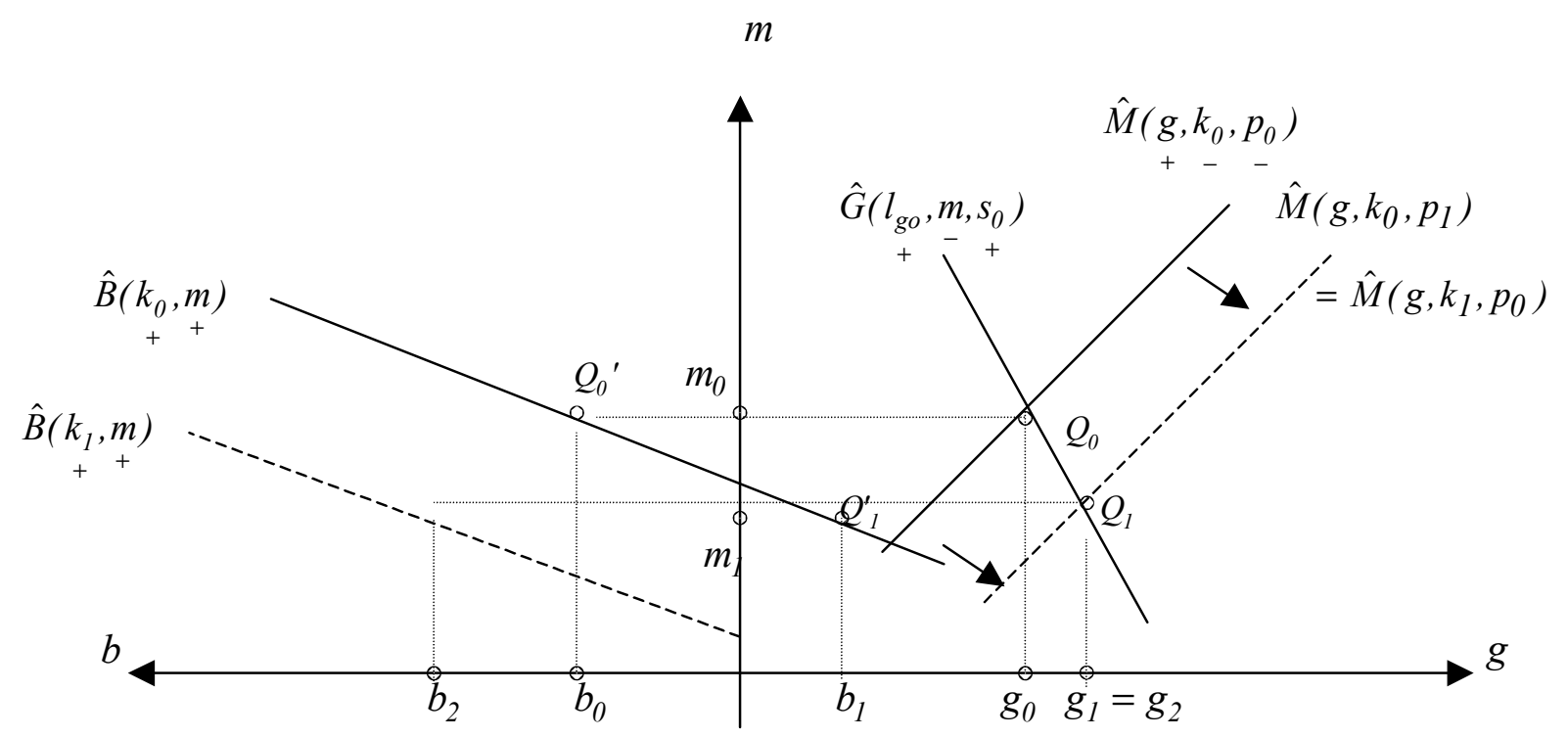

Figure 1: Short-run ecological equilibrium 